\title{
The influence of an external symbol system on number parity representation, or What's odd about 6 ?
}

\author{
WIEBKE IVERSEN \\ Collaborative Research Centre: Media and Cultural Communication, Cologne, Germany \\ HANS-CHRISTOPH NUERK \\ IZKF "BioMat." and RWTH Aachen University, Aachen, Germany \\ and University of Salzburg, Salzburg, Austria \\ LUDWIG JÄGER \\ Collaborative Research Centre: Media and Cultural Communication, Cologne, Germany \\ and RWTH Aachen University, Aachen, Germany \\ and \\ KLAUS WILLMES \\ IZKF "BioMat." and RWTH Aachen University, Aachen, Germany
}

\begin{abstract}
The aim of this study was to investigate whether language-specific properties influence mental number processing. German Sign Language (DGS) numbers differ from those in spoken German not only in terms of modality but also in their basic language structure. A group of 20 congenitally deaf German signers participated in a number parity (odd/even) judgment task with DGS and printed German number words. The results indicated that two-handed DGS number signs are processed in a decomposed way. This language-specific effect also generalized to another linguistic number notation, German number words, but not to Arabic digit notation. These differences are discussed with respect to two possible routes to number parity.
\end{abstract}

Human number processing is assumed to rest on two dissociable mental systems: a language-specific symbol system for exact numerical operations, and a nonsymbolic representation of approximate quantities (see Dehaene, Spelke, Pinel, Stanescu, \& Tsivkin, 1999; Lemer, Dehaene, Spelke, \& Cohen, 2003). Those basic assumptions about the general cognitive architecture of numerical cognition should be valid for deaf and hearing populations alike. Consequently, language-specific properties of German Sign Language (DGS), which is the natural language of the German deaf community, should exclusively affect

This research was supported by funding from the Deutsche Forschungsgemeinschaft supporting W.I. (SFB/FK 427; A1) and H.-C.N. (DFG KFO 112, TP2) and by the Marie Curie Research Training Network (Number and Brain Development, NUMBRA), Proposal 504927. Additional support was provided by the Interdisciplinary Centre for Clinical Research (IZKF “BioMat.") at the Medical Faculty, RWTH Aachen University (Project TV N33). We thank Isa Werth for signing the instructions and test stimuli, as well as Horst Sieprath, Frank Domahs, and Ege Karar for helpful discussions. Thanks as well to Marc Marschark, Marc Brysbaert, and an anonymous reviewer for helpful remarks. Finally, we thank the members of the German deaf community who participated in the experiments. Correspondence relating to this article may be sent to W. Iversen, Media and Cultural Communication Collaborative Research Centre (SFB/FK 427), University of Cologne, Bernhard-Feilchenfeld-Strasse 11, D-50969 Cologne, Germany (e-mail: wiebke.iversen@uni-koeln.de). those numerical operations that require the activation of language-specific mental number formats.

The DGS number system has a main-base-10, sub-base5 structure (see below). Deaf people are also very familiar with the base-10 Arabic number notation, and they are able to process written base-10 German number words. Thus, German signers not only use three different number symbol systems in daily life (as do hearing people), but also two different number systems. This situation has rarely been studied in number processing research so far. Previous studies focused instead on languages with base-10 counting systems or on languages spoken in small cultural communities that do not use Arabic digits or a comparable symbol system at all (Gordon, 2004; Pica, Lemer, Izard, \& Dehaene, 2004). Comparing the processing of the two different number systems in German signers may provide insights into the interrelations of external symbol structure and basic number representations. Moreover, language-specific effects may shed some light on the role of the external symbol structure in number cognition in general, since there is no reason to assume different basic structures of number cognition for deaf and hearing participants.

The DGS number lexicon consists of five different handshapes for the numbers $1-5$, plus a 0 handshape (see Figure 1). Number sequences are signed from the thumb ("1" handshape) to the pinkie ("4" and " 5 " handshapes) on the dominant hand (or H1). For the DGS numbers 6-10, 

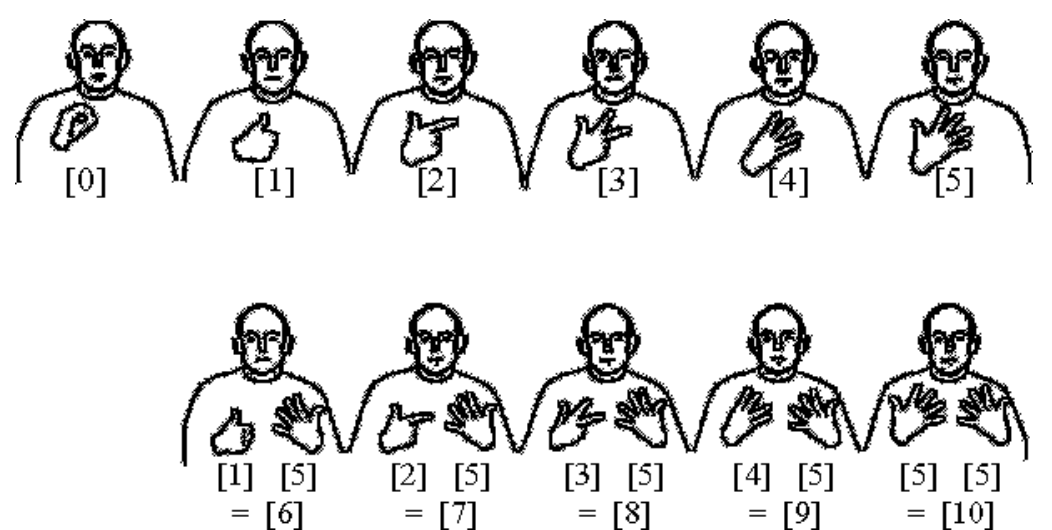

Figure 1. Number signs from 0 to 10 in German Sign Language (DGS). These numbers are performed in a signing space in front of the signer's chest, with the palm toward the signer and with the fingertips in an upward position. For " 1 ," the thumb on the dominant hand (H1) is extended; for " 2 ," the thumb and index finger are extended; for " 3 ," the thumb, index finger, and ring finger are extended; for " 4 ," the index finger, middle finger, ring finger, and pinkie are extended; and for " 5 ," all fingers of $\mathrm{H} 1$ are extended. There are some regional differences for DGS numbers in Germany, but all have in common a one-to-one correspondence between the numerosity of fingers extended in space and the numerical value. All two-handed number signs are combinations of the " 5 " handshape on the nondominant hand (H2) and the " 1, " " 2, ," 3 ," " 4 ," or " 5 " handshape on the dominant hand. Only the numbers 2-9 were included in our analyses.

the appropriate $\mathrm{H} 1$ handshape is combined with a constant "5" handshape on the nondominant hand (H2). If a signer is holding something in one hand, he or she can produce a two-handed sign by signing the $\mathrm{H} 1$ part completely while only sketching the " 5 " handshape on $\mathrm{H} 2$, for instance with a slight movement of the arm.

According to the representational analysis of numeration systems by Zhang and Norman (1995), the DGS number system can be classified as a base-10, sub-base-5 system, as opposed to the purely base-10 system of Arabic number symbols. Zhang and Norman suggested that the structure of a symbolic system influences cognitive processing even in basic (number) tasks. In particular, the sub-base-5 system in DGS may affect the processing of number parity retrieval, since the parity status of the dominant handshape (H1 parity) in two-handed signs $(6-10)$ is different from the parity status of the complete numerical expression (H1 and $\mathrm{H} 2$ parity). For instance, the even numbers 6 and 8 are composed of the odd number " 1 " and " 3 " handshapes (respectively) on $\mathrm{H} 1$ and the odd number " 5 " handshape on H2. Odd two-handed numbers (e.g., 7 or 9) are a combination of an odd number component (the " 5 " handshape) and an even one (the " 2 " or " 4 " handshape).

In number parity judgment tasks, hearing participants responded relatively faster to smaller numbers with the left-hand key and to larger numbers with the right-hand key (Dehaene, Bossini, \& Giraux, 1993; see also Fias, Brysbaert, Geypens, \& d'Y Yewalle, 1996; Nuerk, Iversen, $\&$ Willmes, 2004). This spatial numerical association of response codes (SNARC) effect was observed irrespective of number parity and notation or of the modality in which the numbers were presented (Nuerk, Wood, \& Willmes, 2005), and it was also present for deaf participants when they judged Arabic digits (Bull, Marschark, \& BlattoVallee, 2005; Iversen, Nuerk, \& Willmes, 2004). These results have been taken to indicate the involvement of a nonsymbolic magnitude representation that functions like a "mental number line," with increasing numbers from the left to the right. Orientation of the number line also seems to be influenced by the direction of writing: Iranian participants (from a right-to-left writing community) who had been living in France (with a left-to-right writing system) for a relatively short time tended to show a less pronounced SNARC effect than did Iranian participants who had been staying in France for a longer time (Dehaene et al., 1993). Since there is no conventional written form of DGS, orientation of the number line in deaf signers might instead be linked to the direction of number sequences articulated in space.

Another stimulus-response congruency effect in number parity tasks has been reported by Nuerk et al. (2004). German speakers identified odd numbers relatively faster when responding with the left-hand key, but the opposite was true for even numbers. This linguistic markedness association of response codes (MARC) effect indicates that congruent associations among marked (left-odd) and unmarked (right-even) lexical entities lead to faster responses than do incongruent associations (left-even, right-odd).

In a more recent study, German deaf signers were asked to judge the parity of single-digit Arabic numbers (Iversen et al., 2004). The result patterns revealed a significant 
"regular" SNARC effect. There was also a MARC effect, but it was reversed relative to the one for hearing participants: Odd numbers were responded to relatively faster with the right-hand key than were even numbers, and vice versa. Two possible explanations were provided for this effect: The DGS number sign " 1 " is also used emblematically to indicate "good," "okay," and so forth. These unmarked connotations of the prototypical odd number "1" may influence the markedness of the terms odd and even in the context of numbers. On the other hand, the reverse MARC effect could also be related to the fact that the DGS sign "left hand" is expressed by touching the left arm with the right hand. The activity of the opposite hand may influence the linguistic markedness of the concepts "left" and "right." Both explanations relate to a languagespecific impact on linguistic markedness, but not to the DGS sub-base-5 number system.

In the present study, we investigated whether the subbase-5 system has any influence when printed German number words and photos of DGS number signs are used as the stimulus materials. The three main research questions and expectations for this study were as follows:

1. Is there an influence of the sub-base-5 DGS number system on parity retrieval of DGS numbers?

In principle, two different possibilities exist of how two-handed DGS number signs are processed:

Holistic parity processing. Dehaene et al. (1993) assumed that number parity information is retrieved from a semantic store accessible exclusively via the mental visual Arabic number format. This assumption implies that DGS numbers have to be transcoded into the base-10 Arabic number format before any parity information can be retrieved, in which case the two-handed DGS number signs are processed as holistic number magnitudes. That is, the sub-base-5 DGS number structure would have no impact on parity retrieval, and the result pattern for DGS number signs should be very similar to the one observed for Arabic digit notation, a left-hand preference for even numbers (the reverse MARC effect).

Decomposed parity processing. The handshapes in twohanded number signs are processed separately, although they are presented simultaneously. This would be similar to the processing of two-digit Arabic numbers. Dehaene et al. (1993, Experiment 2) found for the Arabic notation that parity judgments were impeded when the parity of the decades digit conflicted with the parity of the unit digit. Since there is incongruity of parity status for all twohanded DGS number signs, the association of response side and parity could be related to the dynamic dominant number component (H1 parity) rather than to the wholenumber parity (H1 and $\mathrm{H} 2$ parity). In that case, the orientation of the MARC effect should differ for one- and two-handed number signs. Since this was not observed for Arabic digits, it would indicate a format-specific route to parity retrieval.

Independent of both possibilities, the sublexical structure of the combined two-handed number signs is more complex than the structure of one-handed number signs. Thus, numbers in the range $2-5$ might be responded to faster than numbers in the range 6-9 (number range effect).

2. Is there an impact of the DGS number system on the processing of written German numbers?

If Dehaene's (1993) hypothesis of parity retrieval via the Arabic number format is correct, the pattern of results for number words should be similar to the one for Arabic numbers. In contrast, an influence of the sub-base-5 number system on the processing of the base- 10 number words would indicate that signers translate German number words into DGS numbers before retrieving number parity.

3. Is there an influence of the visuospatial modality of sign language on the orientation of the mental number line?

As mentioned above, the orientation of the mental number line in deaf signers could be linked to the direction of DGS number sequences articulated in space. The smallto-large orientation of DGS number sequences for righthanders is from right $(1=$ thumb) to left ( 4 and $5=$ pinkie) in the signer's egocentric perspective. The addressee (allocentrically) perceives the same sequence in the opposite direction from left to right, which is the same orientation as in Western writing systems. If the orientation of the mental number line is modulated via the egocentric perspective of sign language, deaf signers might respond faster with the right hand to small numbers and with the left hand to larger numbers. If the allocentric perspective is decisive, a "normal" left-right SNARC effect would be expected.

\section{METHOD}

\section{Participants}

Twenty prelingually deaf signers ( 12 men; mean age 31 years, range 23-47, 19 right-handed) were tested and paid for participation. All of them had also participated in a study on Arabic digits (Iversen et al., 2004). They used DGS as their preferred language, and most of them had learned DGS in kindergarten (3-4 years of age). All participants had normal or corrected-to-normal vision.

\section{Stimuli and Design}

The participants were instructed to decide whether a presented numeral was odd or even by pressing one of two response keys with the left or right index finger.

Printed German number words and photos of DGS number signs in the range $0-9$ were presented, with nine repetitions per digit in separate blocks. After a short break, both blocks were repeated with the opposite hand-parity assignment. The experiment consisted of $2 \times 2$ blocks of 90 trials each. Both experimental parts started with 12 practice trials.

The participants sat approximately $50 \mathrm{~cm}$ away from the monitor. A fixation cross was presented for $300 \mathrm{msec}$ in the middle of the screen, followed by a stimulus presented in the same position until keypress, but for no longer than $1,300 \mathrm{msec}$. The trials were separated by a blank screen for $1,500 \mathrm{msec}$.

All printed numbers were presented in NRC7BIT 0.64 font with the experimental program ERTS (Beringer, 2000). The single-letter size extended to $1.5^{\circ}$ vertical angle and $1.0^{\circ}$ horizontal angle. DGS numbers were shown as colored photographs of the upper half of a deaf person signing a number. The photos were $11 \times 8 \mathrm{~cm}$ (width by height: $300 \times 215$ pixels) on the screen. The signed numbers extended approximately to $1.5^{\circ}$ vertical angle and $1.4^{\circ}$ (one-handed) and $2.2^{\circ}$ (two-handed) horizontal angle. 


\section{RESULTS}

Answers to the numbers 0 and 1 were not included in the analyses reported here. Zero is supposed to have a special status relative to all other numbers (see Brysbaert, 1995; Nuerk et al., 2004), and the DGS number 1 is a polysemic sign (see above).

Incorrect answers were excluded. In addition, reaction times (RTs) less than $200 \mathrm{msec}$ or more than 1,500 msec, as well as RTs beyond \pm 3 standard deviations from the individual mean, were not included in the analyses. Altogether, $4.1 \%$ of the trials for numbers $2-9$ were excluded. ${ }^{1}$

Mean RTs and error rates for each number and response hand in DGS and printed German are presented in Table 1, together with mean RTs and error rates from our previous study with Arabic numbers (Iversen et al., 2004).

\section{SNARC and MARC Effects}

For both number presentations (DGS number signs and printed German), the mean RT difference between response hands (right - left) was entered into two separate multiple linear regression analyses per participant (Fias et al., 1996; Lorch \& Meyers, 1990; Nuerk et al., 2004), with magnitude $(2,3,4,5,6,7,8$, or 9$)$, parity $(1=$ even vs. $-1=$ odd), and parity of the handshape on the dominant hand ( $\mathrm{H} 1$ parity; $1=$ even vs. $-1=$ odd) as predictors. A significant SNARC effect slope was obtained over participants (random effects) for DGS numbers [6 msec per number; $t(19)=-2.42, p<.05]$, as well as a MARC effect for $\mathrm{H} 1$ parity $[b=13.19 \mathrm{msec} ; t(19)=2.39, p<$ $.05]$ but none for $\mathrm{H} 1$ and $\mathrm{H} 2$ parity $[b=-7.77 \mathrm{msec}$; $t(19)=-0.83, p=.42]$. The positive regression weight for $\mathrm{H} 1$ parity indicates a relative left-hand preference even for $\mathrm{H} 1$ handshapes. In a previous study with German hearing participants, a right-even and left-odd preference was found (Nuerk et al., 2004). Thus, the MARC effect for onehanded number signs (H1 representations) in deaf signers was reversed in comparison with the hearing population. For the two-handed number signs 6-9, the reverse MARC effect for $\mathrm{H} 1$ handshapes was again reversed $(\mathrm{H} 1$ and $\mathrm{H} 2$ representations), resulting in the "regular" parity-response hand association found in hearing participants.

For printed German number words, the results were very similar. Again, the random-effects regression analysis yielded a significant SNARC slope $[b=5 \mathrm{msec}$ per number; $t(19)=-3.07, p<.01]$ and a significant MARC effect for $\mathrm{H} 1$ parity $[b=7 \mathrm{msec} ; t(19)=2.06$, $p=.05]$, but no indication emerged of a significant effect for whole-number parity $[b=-0.71 \mathrm{msec} ; t(19)=$ $20.09, p=.93]$.

\section{Number Range Effect}

To examine a possible effect of number range, we conducted a $2 \times 2 \times 2$ repeated measures ANOVA for the mean RTs of the number ranges 2-5 and 6-9 for each response side and participant. The factors number presentation (printed German vs. DGS numbers), response side (left vs. right), and number range (small [2-5] vs. large [6-9]) were included. All factors revealed significant main effects. Printed number words were responded to $81 \mathrm{msec}$ faster than DGS numbers [number words, $545 \mathrm{msec}$; DGS numbers, $626 \mathrm{msec} ; F(1,19)=99.17$, $p<.001]$, indicating longer encoding times for the more complex DGS number photos than for the printed words. Responses with the right-hand response key (574 msec) were faster than those with the left-hand key (596 msec) $[F(1,19)=13.48, p<.01]$, and numbers in the smaller range were responded to $19 \mathrm{msec}$ faster than numbers in the larger range $[F(1,19)=23.20, p<.001]$. There was also a significant interaction of response side and number

Table 1

Mean Response Times (RT, in Milliseconds) and Mean Error Rates (\%) for Each Response Side and Number $(n=20)$ for DGS Numbers and Written German Numbers, As Well As the Results for Arabic Numbers From a Previous Study (Iversen et al., 2004)

\begin{tabular}{|c|c|c|c|c|c|c|c|c|c|}
\hline Response Side & Measure & 2 & 3 & 4 & 5 & 6 & 7 & 8 & 9 \\
\hline \multicolumn{10}{|l|}{ DGS Numbers } \\
\hline \multirow[t]{2}{*}{ Left } & RT & 594 & 638 & 619 & 633 & 651 & 646 & 666 & 663 \\
\hline & Error rate & 4 & 7 & 2 & 6 & 3 & 2 & 6 & 4 \\
\hline \multirow[t]{2}{*}{ Right } & RT & 595 & 614 & 606 & 611 & 609 & 628 & 619 & 622 \\
\hline & Error rate & 2 & 2 & 2 & 1 & 1 & 1 & 1 & 4 \\
\hline \multirow[t]{2}{*}{ Right - left } & RT & 1 & -24 & -14 & -22 & -42 & -17 & -46 & -41 \\
\hline & Error rate & -2 & -5 & 0 & -5 & -2 & -1 & -4 & 0 \\
\hline \multicolumn{10}{|c|}{ Written German Number Words } \\
\hline \multirow[t]{2}{*}{ Left } & RT & 516 & 564 & 536 & 553 & 561 & 562 & 555 & 587 \\
\hline & Error rate & 3 & 4 & 3 & 5 & 3 & 2 & 4 & 8 \\
\hline \multirow[t]{2}{*}{ Right } & RT & 527 & 551 & 527 & 530 & 532 & 557 & 519 & 550 \\
\hline & Error rate & 2 & 2 & 3 & 1 & 3 & 1 & 1 & 3 \\
\hline \multirow[t]{2}{*}{ Right - left } & RT & 11 & -13 & -9 & -23 & -29 & -5 & -36 & -37 \\
\hline & Error rate & -1 & -2 & 0 & -4 & 0 & -1 & -3 & -5 \\
\hline \multicolumn{10}{|l|}{ Arabic Digits } \\
\hline \multirow[t]{2}{*}{ Left } & RT & 473 & 517 & 485 & 541 & 509 & 523 & 520 & 546 \\
\hline & Error rate & 1 & 4 & 2 & 2 & 6 & 4 & 4 & 6 \\
\hline \multirow[t]{2}{*}{ Right } & RT & 498 & 506 & 496 & 506 & 497 & 483 & 489 & 507 \\
\hline & Error rate & 3 & 3 & 3 & 2 & 2 & 1 & 1 & 1 \\
\hline \multirow[t]{2}{*}{ Right - left } & RT & 25 & -11 & 10 & -35 & -12 & -40 & -30 & -39 \\
\hline & Error rate & 2 & -1 & 2 & 0 & -3 & -3 & -3 & -4 \\
\hline
\end{tabular}


range $[F(1,19)=9.06, p<.01]$, indicating that the rightminus left-hand key difference was smaller for numbers in the $2-5$ range than in the $6-9$ range (SNARC effect). ${ }^{2}$

\section{DISCUSSION}

Results will be discussed with regard to the three questions from the introduction before we conclude with a proposal about two possible routes to number parity retrieval.

\section{Influence of the Sub-Base-5 DGS Number System}

The pattern of results for the MARC effect provides clear answers to Questions 1 and 2 from the introduction. Parity is processed in a decomposed way, not holistically via the internal Arabic number representation. This finding highlights the importance of the external symbolic sub-base-5 system for basic number processing. This is also true for number words, indicating that the external

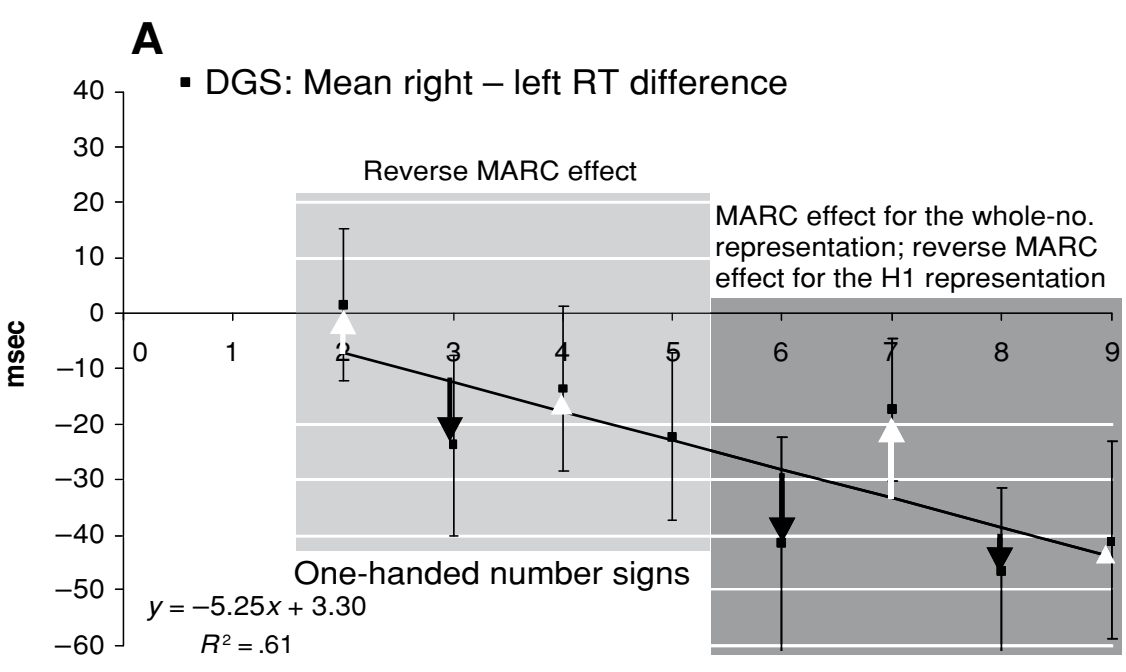

Two-handed number signs

B

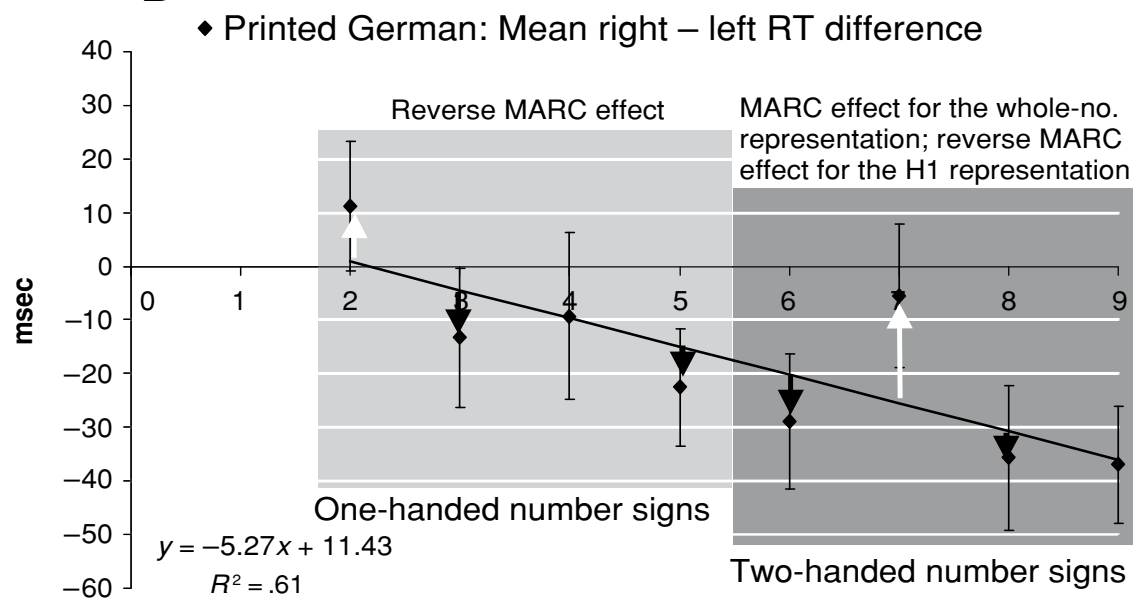

Figure 2. SNARC and MARC effects for (A) German number signs and (B) number words. Mean RT differences between response keys (right minus left) were computed for each numeral in both number formats. On average, answers were given faster with the rightthan with the left-hand key, except with the written number two. The decreasing values of the difference between right- and left-hand keys with increasing numerical magnitude indicate a SNARC effect. This trend is shown in the regression line. There are systematic deviations from the regression line that are positive for most of the even numbers in the range 2-5 (white arrows) and negative for most of the odd numbers in this range (black arrows). This indicates a reversed MARC effect (relative to hearing participants) for both one-handed DGS number signs and the corresponding printed number words. This reversed MARC effect was again reversed for two-handed number signs and printed number words from 6 to 9. 
symbolic sub-base-5 structure influences internal number representations even when this external structure is not transparent in the stimulus material.

The most important result for the MARC effect was that the parity status of the handshape on the dominant hand ( $\mathrm{H} 1$ parity) is a better predictor of the RT pattern than is the parity of the complete number expression, for both DGS and printed number words. For numbers in the range $2-5$, we found a relative RT advantage in the odd-right, even-left condition. This MARC effect is analogous to the one we have found for Arabic digit notation from 2 to 9 in the German deaf group (Iversen et al., 2004), but it is the reverse of result patterns reported for a German hearing group (Nuerk et al., 2004). In the present study, the reverse MARC effect was again reversed for two-handed number signs and the corresponding printed German numbers, resulting in the "regular" parity-response hand association found in hearing participants. For those two-handed numbers, there was a relative RT advantage in the odd-left, even-right condition (see Figures $2 \mathrm{~A}$ and $2 \mathrm{~B}$ ).

Apparently, the handshape of the dominant hand was more relevant for a response (i.e., the judgment of wholenumber parity) than the whole-number sign composed of $\mathrm{H} 1$ and $\mathrm{H} 2$ in two-handed signs. Similar result patterns for printed number words and DGS numbers indicate that base-10 written German numbers are processed via a mental sub-base-5 number format. Signers may encode printed German numbers via an "inner signing" of the corresponding DGS number. ${ }^{3}$

The observation of a small but significant effect of the number range also supports the notion of an influence of sublexical complexity with respect to Questions 1 and 2 . Numbers in the smaller range $(2-5)$ were responded to faster than numbers in the larger range (6-9). A number range effect has not been found for hearing Germans for any notation in number parity tasks (see, e.g., Nuerk et al., 2005), nor for deaf participants judging Arabic digits (Iversen et al., 2004). However, sign frequency or sign length could alternatively account for the effect, since each $\mathrm{H} 1$ number handshape is also part of a two-handed number sign. In sum, the combination of a number range effect and the results regarding the MARC effect are more in line with the decomposed parity processing hypothesis than with a holistic notion of linguistic number parity retrieval.

\section{Orientation of the Mental Number Line}

German deaf signers responded relatively faster with the left hand to smaller numbers and with the right hand to larger numbers. This "normal" SNARC effect provides an answer to our introductory Question 3, since it indicates that the orientation of the mental number line is not modulated by the signer's egocentric perspective of the DGS number sign sequence. We also did not find any influence of the DGS sub-base-5 number system on the spatial

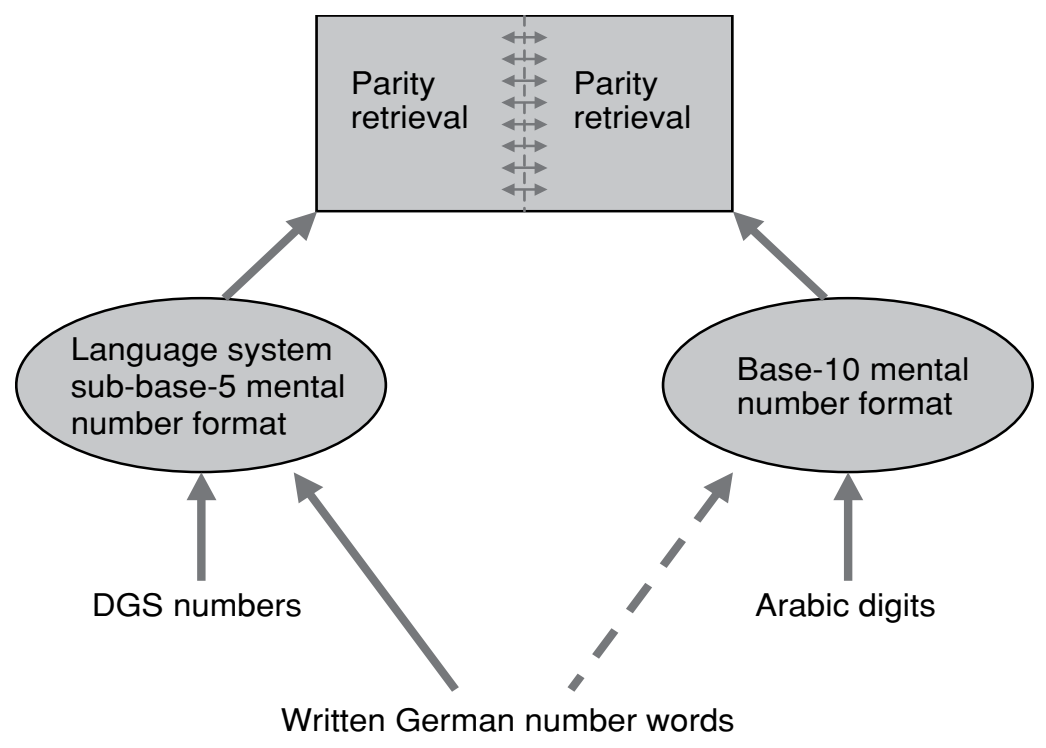

Figure 3. Sketch of two possible routes to number parity. Linguistic number signs access parity information via a language-specific lexical route. Thus, language-specific properties influence the odd/even decision. Arabic digits are assumed to activate a second, symbol-specific route to semantic number parity information. Both modes of access are interrelated, but access is different. Printed German number words may activate both routes, but the languagespecific route seems to be dominant. The dashed arrow indicates that this route might be activated simultaneously. 
orientation of the mental number magnitude representation. This result corroborates the general assumption of a symbol-independent mental number magnitude system.

\section{Two Routes to Parity?}

Similar to the two routes (semantic and asemantic) to number magnitude proposed by Fias (2001) for word numerals, there might also be two different routes to number parity (see Figure 3). These routes are dependent on the input number form. DGS numbers might access number parity information via a language-specific route. Thus, sub-base-5 DGS numbers are not transcoded into a base10 mental number format before number parity gets retrieved. Arabic digits, on the other hand, may activate number parity status via number-specific access, implying that base-10 Arabic numbers are also not transcoded into a mental sub-base-5 number format. Both routes to number parity representation might be interlinked. The impact of the sub-base-5 number structure on the results for written number words indicates that those numbers mainly activate the language-specific route. However, written words might simultaneously activate the second route. This model of parity retrieval is still highly speculative, since German signers are the first group for whom a language-specific influence on number parity retrieval has been observed.

\section{Conclusions}

The results of the present study indicate that the DGS sub-base-5 number system has (1) an impact on parity retrieval of DGS numbers and (2) a similar impact on written German number words as a sub-base-5-independent input notation. However, that system has (3) no impact on the mental number line for any notation, nor (4) any impact on parity retrieval in Arabic notation (Iversen et al., 2004).

These results are in line with the general assumption of two different mental number systems in number cognition. Since the pattern of results for linguistic numbers differed from the one for Arabic digit notation, we have suggested that there might be two different, lexical and semantic routes to parity retrieval. The language-specific counting sequence of DGS differs from the one in aural German, but all other number symbol systems are used by both deaf and hearing persons. Thus, the two routes to parity retrieval might also be valid for the hearing population. Influences of the external symbol system on mental number processes may have been underestimated so far, since virtually all number systems studied share a base- 10 structure with the Arabic notation. In that case, the similarity of patterns of results might be related to the similarity of the structure of the external symbol systems rather than to the similarity of mental structures.

\section{REFERENCES}

Beringer, J. (2000). Experimental Run Time System [Computer software]. Frankfurt am Main: BeriSoft.

BRysbaerT, M. (1995). Arabic number reading: On the nature of the numerical scale and the origin of phonological recoding. Journal of Experimental Psychology: General, 124, 434-452.

Bull, R., Marschark, M., \& Blatto-Vallee, G. (2005). SNARC hunting: Examining number representation in deaf students. Learning \& Individual Differences, 15, 223-236.

Dehaene, S., Bossini, S., \& Giraux, P. (1993). The mental representation of parity and number magnitude. Journal of Experimental Psychology: General, 122, 371-396.

Dehaene, S., Spelke, E., Pinel, P., Stanescu, R., \& Tsivkin, S. (1999). Sources of mathematical thinking: Behavioral and brainimaging evidence. Science, 284, 970-974.

FIAS, W. (2001). Two routes for the processing of verbal numbers: Evidence from the SNARC effect. Psychological Research, 65, 250-259.

Fias, W., Brysbaert, M., Geypens, F., \& D'Ydewalle, G. (1996). The importance of magnitude information in numerical processing: Evidence from the SNARC-effect. Mathematical Cognition, 2, 95-110.

Gordon, P. (2004). Numerical cognition without words: Evidence from Amazonia. Science, 306, 496-499.

Iversen, W., Nuerk, H.-C., \& Willmes, K. (2004). Do signers think differently? The processing of number parity in deaf participants. Cortex, 40, 176-178.

Lemer, C., Dehaene, S., Spelke, E., \& Cohen, L. (2003). Approximate quantities and exact number words: Dissociable systems. Neuropsychologia, 41, 1942-1958.

LORCH, R. F., JR., \& Myers, J. L. (1990). Regression analyses of repeated measures data in cognitive research. Journal of Experimental Psychology: Learning, Memory, \& Cognition, 16, 149-157.

NuERK, H.-C., IVERSEN, W., \& WILLMES, K. (2004). Notational modulation of the SNARC and the MARC (linguistic markedness of response codes) effect. Quarterly Journal of Experimental Psychology, 57A, 835-863.

Nuerk, H.-C., Wood, G., \& Willmes, K. (2005). The universal SNARC effect: The association between number magnitude and space is amodal. Experimental Psychology, 52, 187-194.

Pica, P., Lemer, C., Izard, V., \& Dehaene, S. (2004). Exact and approximate arithmetic in an Amazonian indigene group. Science, 306, 499-503.

Zhang, J., \& Norman, D. A. (1995). A representational analysis of numeration systems. Cognition, 57, 271-295.

\section{NOTES}

1. All analyses were also conducted with the left-handed participant excluded. The results did not differ.

2. The results of the identical ANOVA for Arabic digits were response side, $F(1,19)=14.96, p=.001$; number range, $F(1,19)=1.81, p>$ .15 ; and response side $\times$ number range, $F(1,19)=11.49, p<.01$.

3. There is one alternative explanation for the reverse MARC effect present only for one-handed numbers. Numbers are assumed to be represented on the mental number line in a logarithmically compressed manner. Thus, the spatial distance between the smaller, one-handed number signs on the mental number line is larger than the distance between the larger, two-handed numbers. The tendency to respond faster to smaller numbers with the left hand could be more pronounced for smaller numbers than for larger numbers. Therefore, the small even number 2 is responded to relatively faster with the left hand than is the odd but larger number 3 . In this case, the SNARC effect (relative right-hand preference for small numbers) might outweigh a normal MARC effect (relative right-hand preference for even numbers) for smaller numbers, and a normal MARC effect might outweigh a SNARC effect for larger numbers. The reverse MARC effect that is again reversed for two-handed number signs could then have nothing to do with the language-specific properties of DGS. Because a reverse MARC effect was never observed for hearing participants, nor a "regular" MARC effect for numbers in the 6-9 range when the German deaf group judged Arabic digits (Iversen et al., 2004), this possibility does not seem convincing in light of the previous data.

(Manuscript received May 30, 2005; revision accepted for publication November 30, 2005.) 